\title{
Studies on the phrenic nerve and diaphragmatic plexus in rabbits and gerbils
}

\author{
ROBERT MITCHELL and ANDREW S. WILSON \\ Department of Anatomy, University of Western Ontario, London 72, Canada
}

\begin{abstract}
Studies on nerve fibres within the phrenic nerve and in the diaphragmatic plexus were carried out in one species, the gerbil, a small desert rodent. The resistance of this small mammal to surgical insult enabled experimental intrathoracic transection of the phrenic nerve to be carried out so that the results in 10 animals could be observed within the diaphragmatic plexus, the phrenic nerve, and the phrenic nucleus. The findings in the diaphragmatic plexus and in the nerve trunk strongly resemble corresponding results for other animals with regard to 'persisting' fibres. The phrenic nucleus, however, is much more extensive in this mammal than has been found in any other species; it is represented within the second to sixth cervical segment, its rostral extremity occupying the intermediolateral part of the spinal grey matter. The possible functional significance of these findings is discussed in the light of the mixed functions of the diaphragm, either voluntary or involuntary.
\end{abstract}

An extensive search of the literature reveals that almost all of the relevant studies during the past century have considered only small circumscribed aspects of diaphragmatic nerve supply. Thus, while Kohnstamm (1898), Hirako (1928), Keswani, Groat, and Hollinshead (1954) and Samuel and Warwick (1955) gave their full attention to the 'phrenic nucleus', Sherrington (1894), Kidd (1911), and Botha (1957) placed more emphasis on the peripheral features of diaphragmatic innervation. Although limited conclusions can be drawn from these records, species differences in the various studies preclude direct comparisons, thus diminishing the collective significance of the results.

These criticisms have been forestalled in this investigation by applying reliable methods used in cats and rodents (Wilson, 1968) to one species, the gerbil. The principal aim here is to obtain a more comprehensive overview of the phrenic nerve-diaphragm apparatus in gerbils by including spinal cord connexions, fibre spectrum of phrenic nerve, and structural features of the diaphragmatic plexus.

\section{MATERIAL AND METHODS}

INVESTIGATIONS IN NORMAL ANIMALS

TOPOGRAPHICAL STUDIES Three adult gerbils, killed with a lethal dose of intraperitoneal sodium pentobarbitone, were perfused with formol saline and immersed in formalin $5 \%$ for several days. The chest cage was opened widely and the phrenic nerve on each side was traced centripetally from its conspicuous diaphragmatic extremity to its source. In each case the level of origin in the cervical spinal segments was determined by reference to the bodies of adjacent cervical vertebrae.

HISTOLOGICAL STUDIES In three gerbils and three rabbits (all adults), selected at random without reference to sex or feeding habits, anaesthesia was induced by intraperitoneal injection of sodium pentobarbitone, $48 \mathrm{mg} / \mathrm{kg}$, and each animal was perfused through the abdominal aorta with formol saline $10 \%$ until the return flow through the inferior vena cava was clear. This method of fixation was standard for all histological studies which, from this point onwards, varied according to the tissue under investigation.

Myelinated fibres of the phrenic nerve After thorough washing in several changes of distilled water, 2-mm lengths of phrenic nerve taken from central, intermediate, and peripheral levels were immersed (in the dark) in a $1 \%$ solution of osmic acid for 1 to 3 hours, depending on the thickness of the specimen. These were then washed thoroughly, dehydrated, and embedded in wax, and sections, cut at a thickness of $5 \mu$, were mounted and photographed along with a micrometer scale so that accurate magnifications could be calculated.

Studies on the whole diaphragm (a) Silver impregnation: Following formol-saline perfusion, gerbils intended for silver studies on the diaphragmatic plexus 

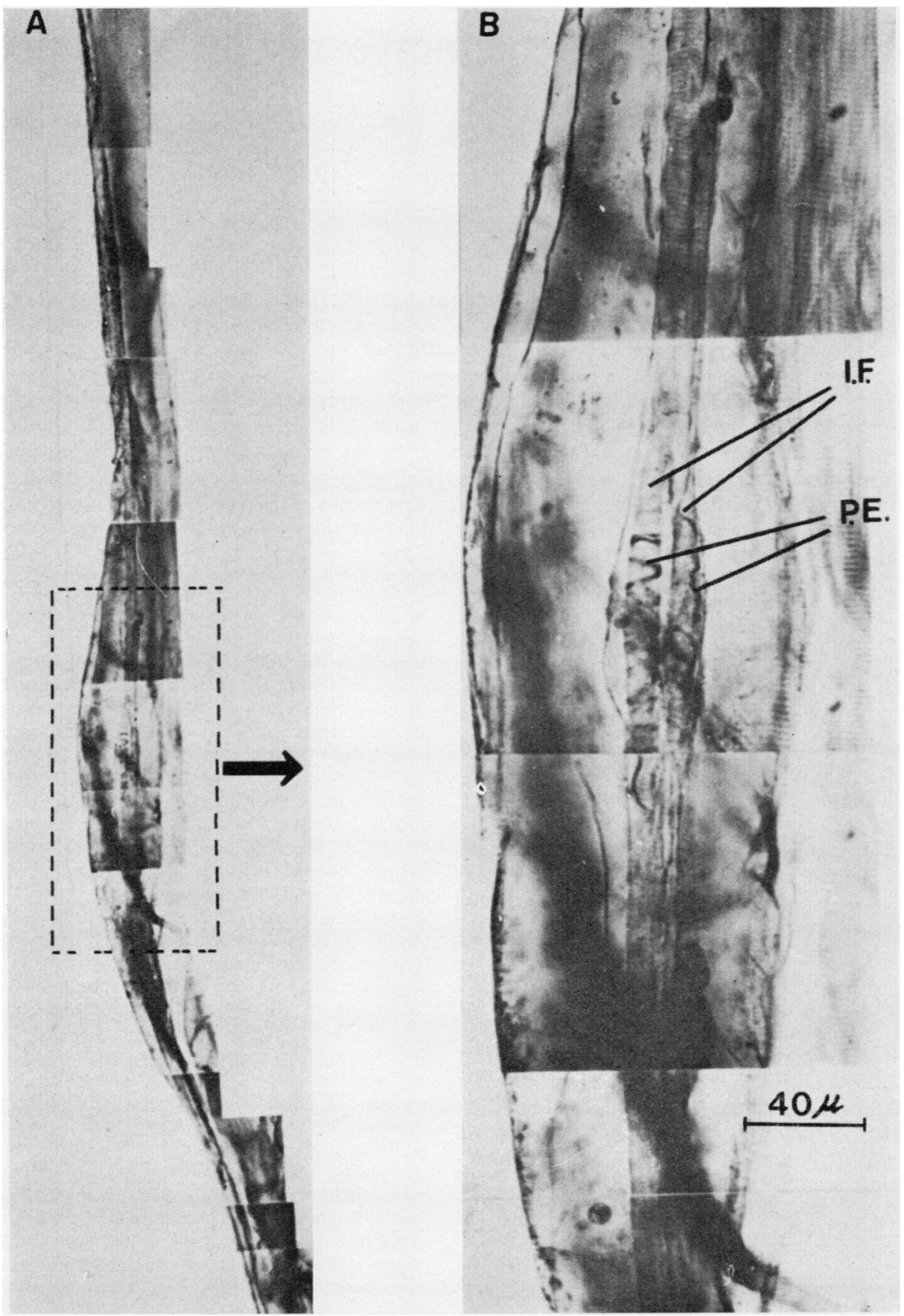

FIG. 1. Represents a muscle spindle within the gerbil diaphragm. Striations can be seen within intrafusal fibres (I.F.) and the primary endings (P.E.) are well defined. The broken line in $A$ indicates the area shown in $B$. 
RABBIT

GERB I L
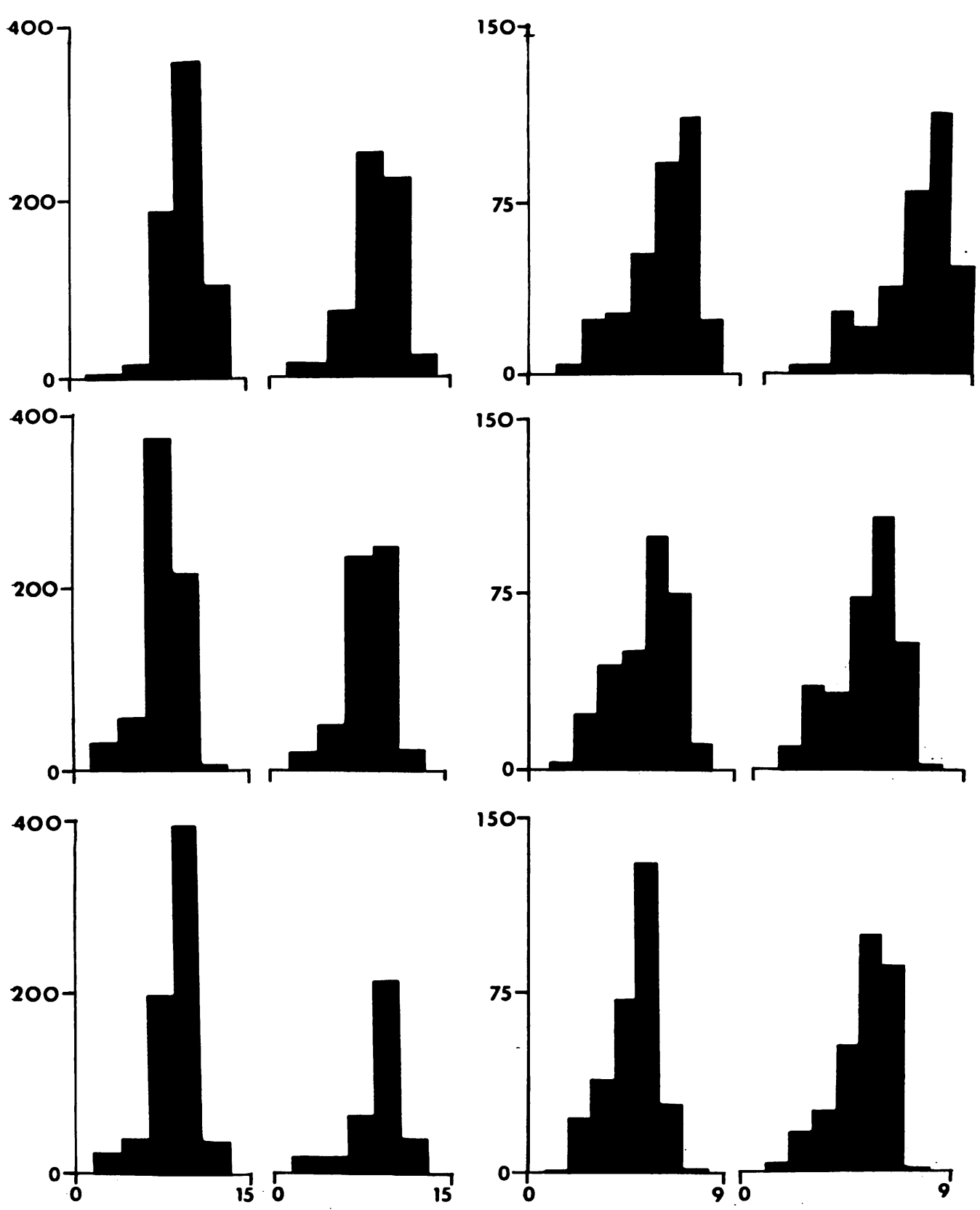

MICRONS

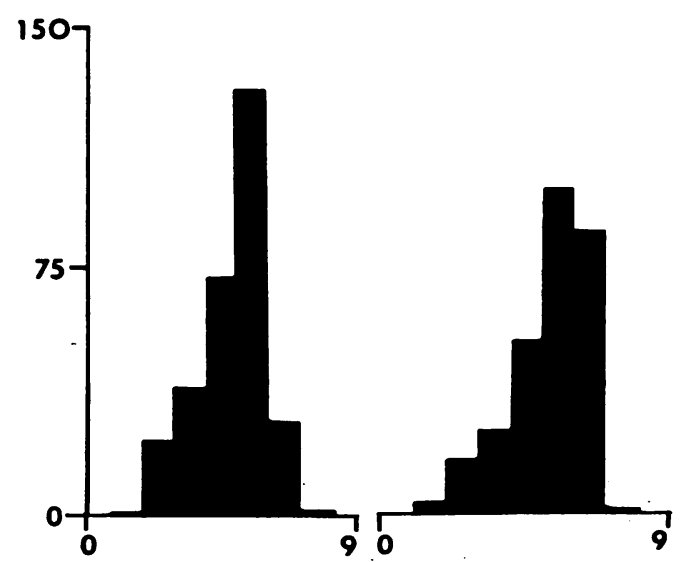

MICRONS

FIG. 2. Histograms illustrating numbers of myelinated fibres (ordinate) within specific ranges of external diameter (abscissa). Upper, middle, and lower profiles represent the fibre population at proximal, middle, and distal levels respectively. 
were stored in $10 \%$ neutral formalin for several days with the thoracic and abdominal cavity widely opened. The diaphragm was removed in one piece and impregnated with silver as described by Garven and Gairns (1952). Longer immersion times in some solutions were necessary because the diaphragm is several times thicker than the tissues for which the method was originally intended.

(b) Gold chloride impregnation: In two gerbils and two rabbits, the diaphragm was removed and impregnated as a whole specimen in the manner described by Gairns (1930). Adequately impregnated specime.1s were examined as a series of 'teased' preparations.

(c) Quantitative methods: Photographs representing the myelinated fibre population at specified levels within the phrenic nerve were reproduced on thin paper, and the size distribution was recorded on a suitably calibrated Zeiss Particle Size Analyser TGZ3. These quantitative data were processed in the computer by subjecting them to a carefully contrived programme of sufficient versatility to be applicable to any peripheral nerve.

\section{INVESTIGATIONS IN EXPERIMENTAL ANIMALS}

SURGICAL PROCEDURE In each animal of a series of eight randomly selected adult gerbils, anaesthesia was induced as described above. The skin was suitably prepared and tracheostomy was carried out at the level of the suprasternal notch. A sterile polythene cannula was inserted to permit insufflation of the lung during subsequent endothoracic manipulation.

The phrenic nerve was exposed through an incision in the seventh intercostal space; the nerve trunk was divided about 8 to $10 \mathrm{~mm}$ rostral to the diaphragm and the chest wound was sutured immediately with the lungs in a state of full inflation. In most animals spontaneous, rhythmic respiration, at twice the preoperative rate, ensued at once but occasionally half a minute of assisted respiration was necessary to maintain the animal. The cannula was removed from the trachea and the skin was repaired.

HISTOLOGICAL METHODS After survival periods of 2 to 21 days, myelinated fibres of the phrenic nerve peripheral to the level of the lesion were demonstrated by the osmium method shown above. Silver impregnation of the diaphragmatic plexus was also carried out according to the method described for normal animals.

Studies on the phrenic nucleus After survival periods of 1 to 28 days individual animals were perfused as before, and after two to three weeks in formalin the upper half of the spinal cord was removed, dehydrated, and embedded in wax so that central and peripheral ends of the specimen could be identified. The 'middle cut' from this specimen, corresponding to cervical segments $C_{3}$ to $C_{7}$, was sectioned serially at a thickness of $10 \mu$ and stained with cresyl violet.

\section{OBSERVATIONS IN NORMAL ANIMALS}

MACROSCOPIC FINDINGS In three gerbils, the phrenic $\overline{\bar{n}}$ nerve was traced to its spinal origin which included $\overrightarrow{\widetilde{\sigma}}$ cervical segments $3,4,5$, and 6 . With the aid of theo dissecting microscope, some local connexions were identified in the region of the thoracic inlet. The fur- $\vec{P}$ ther course and mode of termination of the phrenic. nerves along with the general structural features of $\vec{\omega}$ the diaphragm so closely resemble the arrangements in other mammals that they warrant no further des- $\overrightarrow{\vec{x}}$
cription here.

\section{HISTOLOGICAL FINDINGS}

Receptors in the diaphragm In gerbils, muscle-v spindles were observed in silver-impregnated dia-o phragms which were subsequently teased apart for_ high-power microscopy. These receptor devices were few in number but parts of them were seen in almosto every diaphragm examined. Figure 1 represents thes most complete example seen in the diaphragm, its . overall length being $400 \mu$. It is estimated that no more than three muscle spindles were present in each hemi? diaphragm.

In addition to these receptors, the diaphragmatice plexus contained many nerve fibres of smaller diameter which have no identifiable end organs and mayo therefore be presumed to ramify as free nerve endings? Although some of these fibres occupy paravascularo positions and may be identified as possible autonomic components, many ramify at considerable distances5 from vessels.

Myelinated fibres of the phrenic nerve. To calibrate the Particle Size Analyser for this study a preliminary trial was carried out with rabbit phrenic nerves. Abso lute frequency graphs as produced by the computer comprise size-ranges which are so narrow that it is difficult to discern any difference in the distribution pattern at these separate levels. However, when the computer data are rearranged so that the range of diameter size; is increased in each group (thus reduc은 ing the number of groups) size distribution differencess become more apparent.

Histograms, modified in this way, representing the fibre population at three distinct levels in four phrenior nerves selected at random, from two rabbits and two gerbils, are shown in Figure 2 . It can be seen that the range of diameters is 1.5 to $14 \mu$ in rabbits and 1.250 to $9 \mu$ in gerbils. The graph for rabbits is unimodalo the peak occurring at diameters ranging from 7 to 11 o while, in gerbils, the size distribution is clearly bimodal in one nerve, the peaks occurring at diamete? ranges 2.3 to $3.2 \mu$ and 6.7 to $7.6 \mu$.

With the exception of the rabbit nerve represented in column 2 of Fig. 2 the total numbers of fibres seen to be approximately equal at all three levels in the other nerves. These findings are consistent with results obtained in quantitative studies in other animals of this and previous investigations. Maintenance of these 

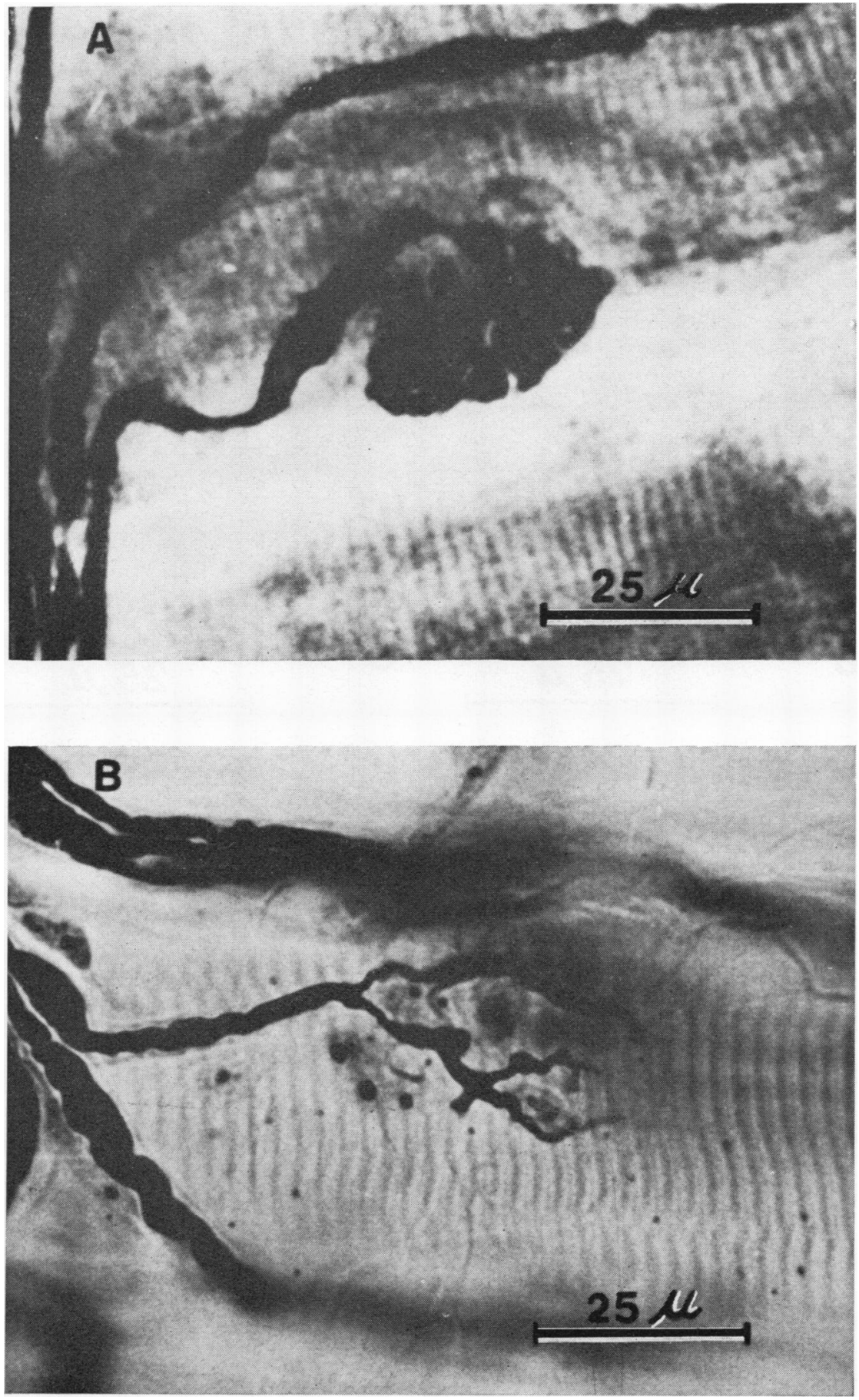

FIG. 3. (A) Motor end plate from a rabbit diaphragm impregnated with gold. (B) Motor end plate from a gerbil diaphragm impregnated with silver. 

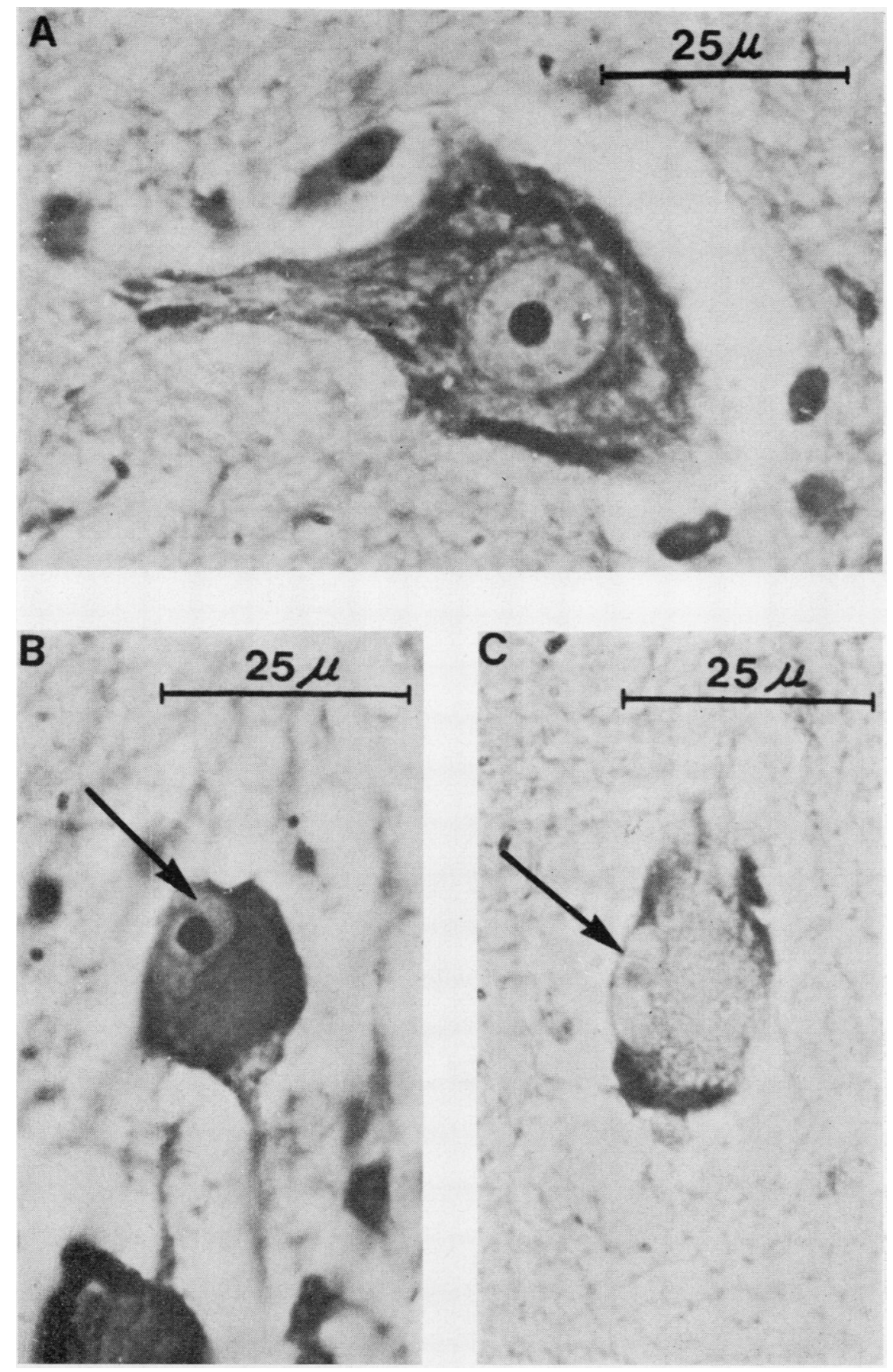

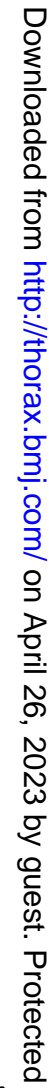

FIG. 4. Chromatolytic neuronal changes in the cervical segments of the spinal cord 21 days after transection of the phrenic nerve within the chest: $(A)$ normal neurone; $(B$ and $C)$ chromatolytic cells showing cytoplasmic granularity and eccentric nucleus (arrow). 
consistent totals may be related to the general shapes of the histograms which indicate a decrease in total numbers of fibres in the 3 to $9 \mu$ category and reciprocal increases in fibres whose diameter range is 9 to $13 \mu$. This pattern of numerical variation is seen in the rabbit nerves and in one gerbil nerve. Closer scrutiny of the right-hand columns in rabbits reveals that the fibres in size-range 11 to $13 \mu$ increase in number between middle and distal levels. This effect is seen also in one gerbil nerve, the opposite being the case in the remaining nerve.

Motor endings in the diaphragm In this part of the investigation, gold chloride methods most successfully demonstrated motor end plates in rabbits while silver nitrate produced the best results in gerbils (Fig. 3). Both methods show a densely impregnated preterminal expansion and an irregularly branching termination in relation to rounded nuclear profiles which are more densely impregnated in the gold preparation (A). Heterogeneous density is evident in the nuclear profiles of the silver preparation (B) and when it is possible to visualize an individual motor terminal, it

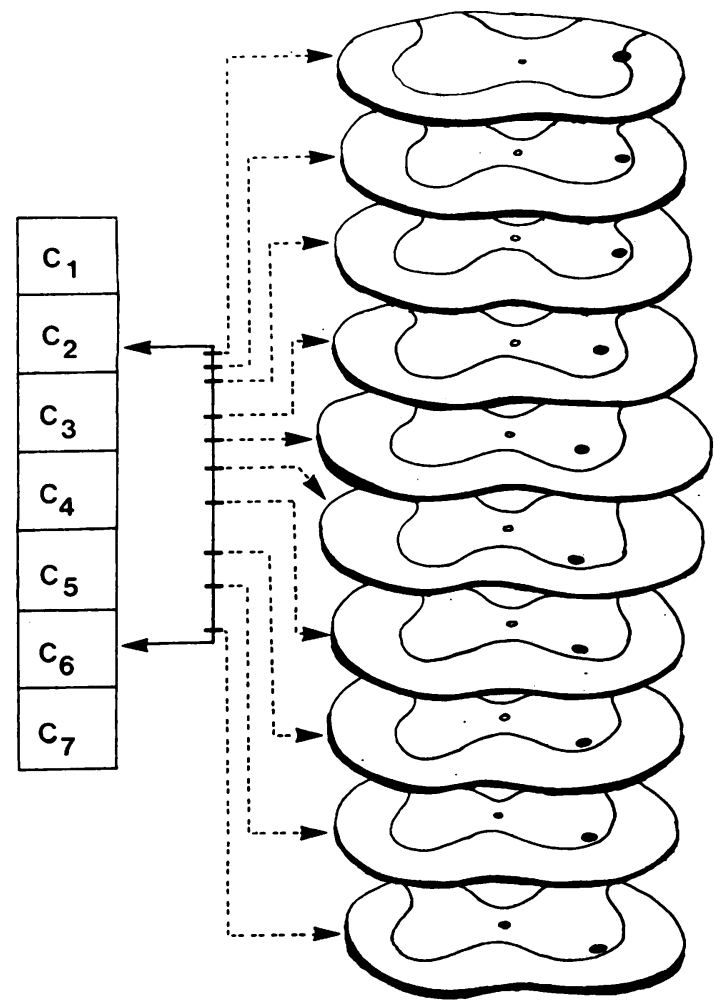

FIG. 5. Illustrates diagrammatically the cervical spinal levels at which chromatolysis (black dots) was seen 21 days after homolateral phrenic transection. is clear that the rabbit and gerbil resemble each other strongly in this respect; these endings are almost equal in size in the two species.

\section{OBSERVATIONS IN EXPERIMENTAL ANIMALS}

THE PHRENIC NUCLEUS In two female gerbils in which intrathoracic phrenic nerve transection had been followed by survival periods of 21 days, the spinal cord was removed and serial sections were prepared for examination with the Nissl stain. Regional chromatolytic changes were seen within cells (Fig. 4) in cervical segments as indicated (Fig. 5). It can be seen that chromatolysis was present in the cervical segments 2 to 6 and that these cells at rostral levels occupied the intermediolateral grey column. This finding was consistent in both animals studied by the serial section method and was confirmed subsequently in a further two gerbils.

CHANGES IN MYELINATED FIBRES PERIPHERAL TO LEVEL OF TRANSECTION These changes were characterized by progressively increasing disruption of the osmiophilic sheaths which began as simple notching of the myelin along with axoplasmic osmiophilia at four days and went on to show almost complete absence of recognizable sheath profiles at 21 days. In no case, however, were all circular osmiophilic profiles totally absent.

CHANGES IN DIAPHRAGMATIC PLEXUS AFTER UNILATERAL TRANSECTION In whole preparations of the diaphragm impregnated with silver there was general evidence of nerve fibre degeneration on the side of the lesion (Fig. 6) but, in every animal examined, there were persisting fibres present. In some specimens, recognizable motor end-plates were observed in diaphragmatic areas otherwise devoid of normal nerve fibres (Fig. 7).

In one animal, 153 days after unilateral phrenic transection, there was evidence of regeneration of axis cylinders. The distal stump of the transected nerve showed a cluster of argyrophilic fibres connected with its proximal end (Fig. 8). These could be traced through the phrenic nerve terminals into the diaphragmatic plexus. Although accurate measurement was impossible, it is estimated that these regenerating fibres were in the diameter range 4 to $10 \mu$.

\section{DISCUSSION}

The selection of the gerbil as the experimental animal for this investigation was prompted by the translucency of its diaphragm and its apparently high resistance to experimental surgical procedures such as thoracotomy. The resemblance of the diaphragm-phrenic nerve apparatus in gerbils to that observed in other small mammals, such as rats and mice (Wilson, 1968), was also considered advan- 

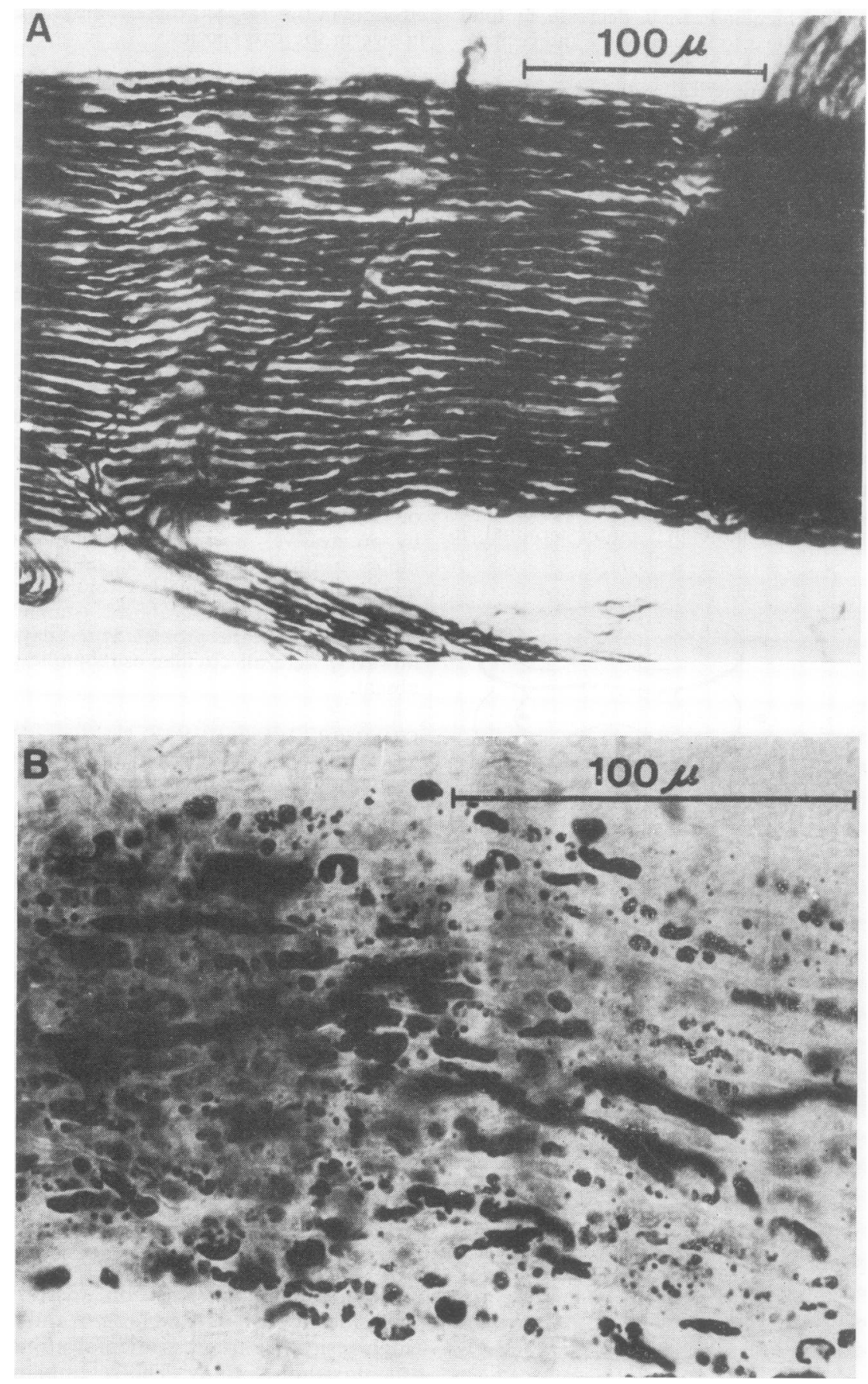

FIG. 6. (A) A fasciculus of nerve fibres within the diaphragm of a normal animal. (B) A comparable fasciculus of the diaphragmatic plexus in which the phrenic nerve had been transected 21 days previously. 

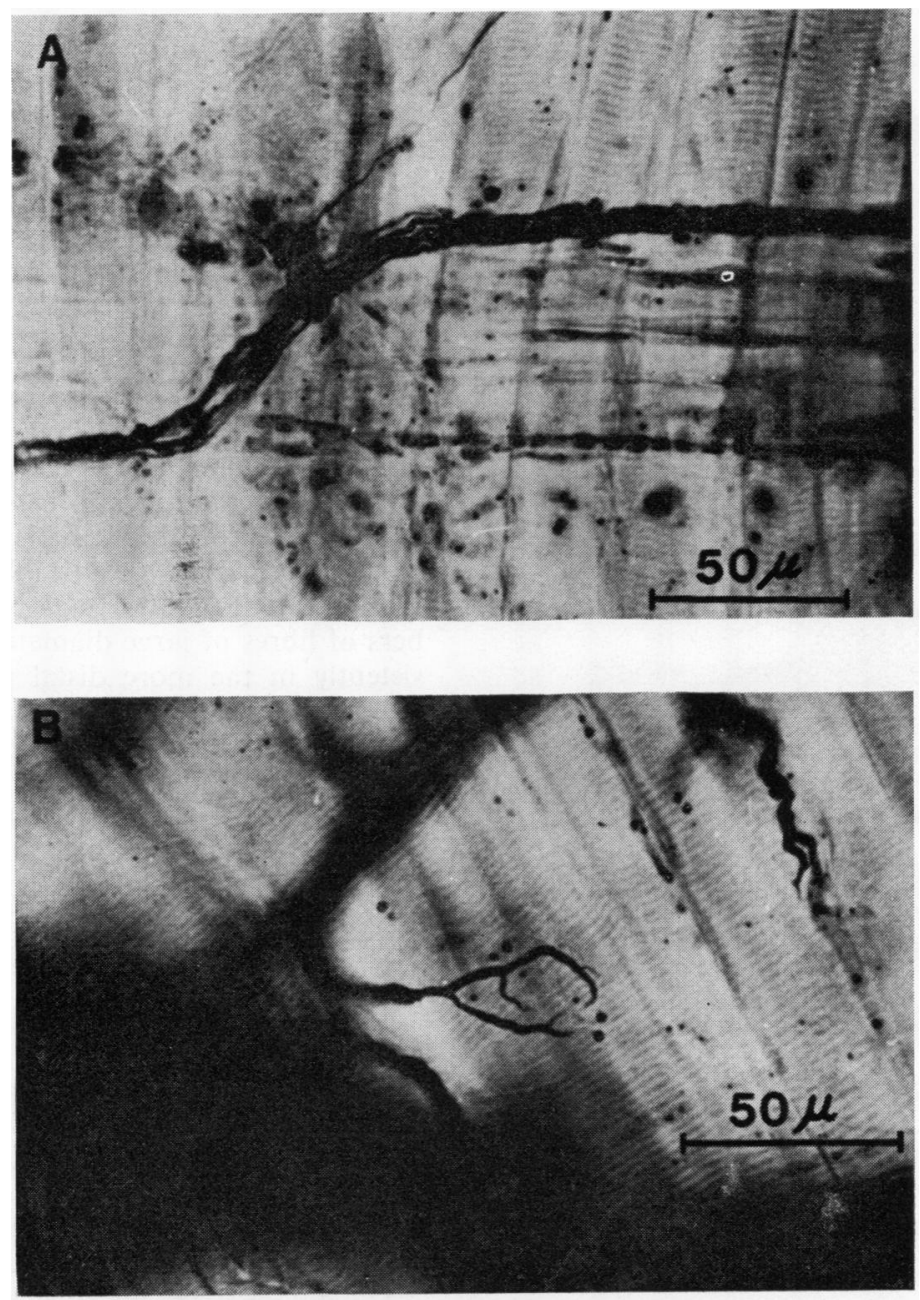

FIG. 7. Neural components in the left hemidiaphragm in which left phrenic transection had been carried out 21 days earlier. (A) A bundle of 'persisting' fibres is seen following the same course as a larger fasciculus in which the nerve fibres are almost totally degenerated. $(B$ and $C)$ Normal motor end plates.

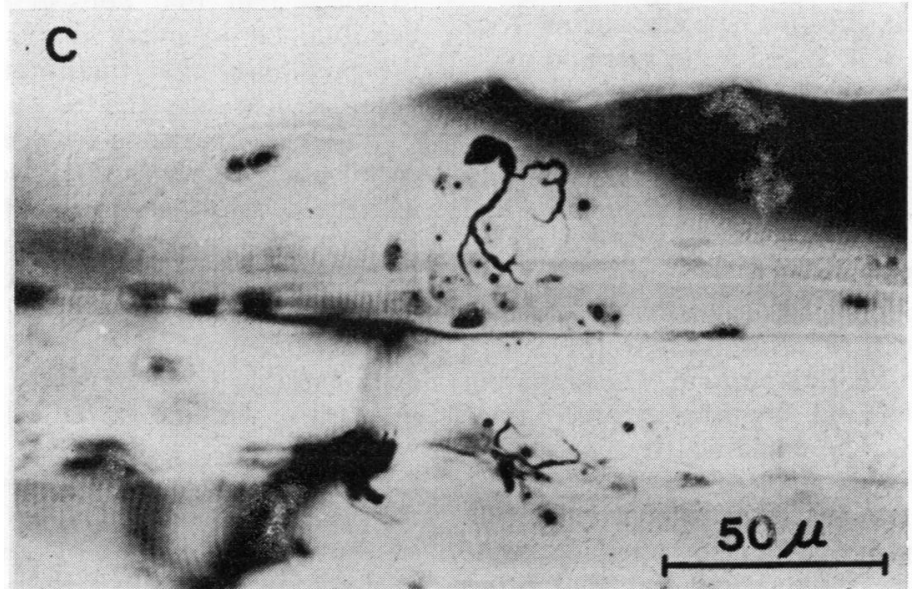




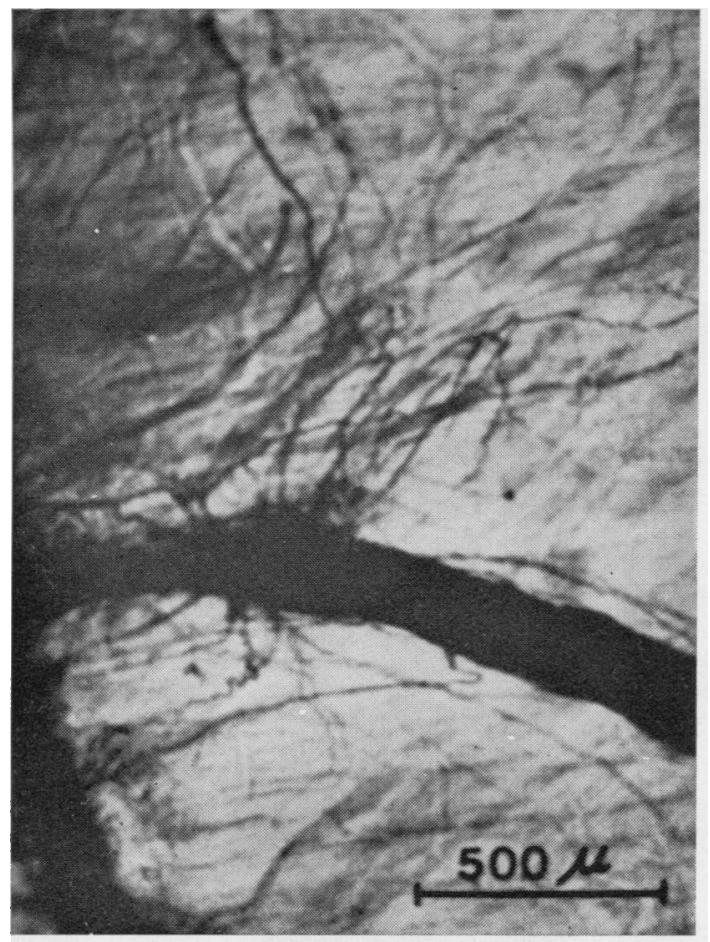

FIG. 8. The 'regeneration' of fibres at the proximal end of the distal stump of a phrenic nerve transected 153 days previously. The nerve stump (directed from right to left) has many argyrophilic fibres converging upon it from many
different directions. ?

tageous for future comparison. One minor difference is the diffuse nature of the phrenic nerve spinal origin which, in this species, spans four cervical segments-3, 4, 5, and 6 : in rats and mice, only the latter two segments appear to participate.

AFFERENT INNERVATION As with other specieś such as rats (Barstad, Kristoffersen, Lilleheil, and Staaland, 1965) and cats (Wilson, 1968), recognizable receptor devices such as muscle spindles are very few in number. It is estimated that, in this species, there can be no more than three such endings within each hemidiaphragm. This apparent discrepancy, emphasized by the profusion of efferent endings, may be explained by postulating that information for control of respiratory muscles can be mediated along the intercostal nerves in which the receptor devices so much resemble their diaphragmatic counterparts and that other afferent pathways, either somatic or autonomic, may exist for diaphragmatic afferent input. It has been confirmed in gerbils that the diaphragmatic $\frac{\text { के }}{0}$ plexus possesses a profusion of nerve fibres of $\frac{}{}$ smaller diameter, 3-10 $\mu$, and that an appreciable $\overline{\bar{\rho}}$ number of these terminate eventually within the $\mathbb{\Phi}$ musculature of the diaphragm.

MYELINATED FIBRES OF THE NERVE TRUNK The finding, in gerbils, that myelinated fibres in the $\vec{\omega}$ nerve trunk show little change in numbers at $\stackrel{\rho}{\vec{*}}$ proximal, middle, and distal levels is in agreement $\vec{x}$ with previous findings in cats (Wilson, 1968). The $N$ maintenance of these totals does not, however, iv seem to be entirely explicable on the same basis $\vec{\oplus}$ as in cats and rabbits. In gerbils, increases in num- $O$ bers of fibres of large diameter are not found con- $\frac{}{3}$ sistently in the more distal portion of the nerve $\vec{z}$ although the near equality of the totals suggests that fibres of medium-small diameter are maintained at a steady level. To explain this, nothing more than intraneural branching would be $\vec{v}$ necessary, whereas, in cats and rabbits, where the $N$ total count is maintained by increasing the numbers of fibres of large diameter, it is necessary to consider at least the possibility of an accession to the nerve in its distal portion.

DinPHRagmatic PLEXUS The diaphragmatic plexus in gerbils is directly comparable with that of mice, rats, and cats; it shows the same preponderance of end-formations which are clearly efferent in nature and resemble motor end-plates seen in silver preparations from the species mentioned.

Difficulties experienced in demonstrating motor end-plates in previous studies were overcome here 3 to the extent that consistent argyrophilia could be obtained in every diaphragm examined, and the possibility that quantitative values might be obtained was investigated.

Direct comparisons between silver-impregnated $\frac{D}{O}$ gerbil diaphragms and those of rabbits treated with gold chloride showed that numbers of end- $\widetilde{N}$ plates varied with the total numbers of myelina- N ted fibres in the nerve trunk.

It may be assumed that, in the diaphragm, nerve fibres which have no specialized ending demonstrable by the present method are afferent in nature. Of the varieties of receptor devices $\stackrel{\oplus}{\rightarrow}$ which have been described (Lanza, 1958), only 0 the muscle spindle was observed in this study, the most complete one coming from the crural zone of the diaphragm and measuring $400 \mu \times 90 \mu \mathbb{\nabla}$ at its longest and widest points respectively; a muscle spindle from gerbil intercostal musculature was almost identical in size and shape. 
EXPERIMENTAL STUDIES The effects of phrenic nerve transection within the chest in gerbils resemble very strongly those found in cats, rats, and mice (Wilson, 1968). Persisting fibres within the hemidiaphragm and subtotal degeneration of myelin sheaths on the same side as the lesion, coupled with some evidence of nerve fibre degeneration in the contralateral hemidiaphragm, suggest collectively that there may be crossing over of nerve fibres in the anterior mediastinum. If this connexion were made rostral to the level of the lesion it would account for persisting sheaths in the nerve trunk and the somewhat unexpected intradiaphragmatic observations just mentioned.

One possibility that still remains is that these persisting processes represent the fibres which have entered the phrenic distribution within the diaphragm from an extraphrenic source. This was postulated to account for the corresponding findings in cats (Wilson, 1968) but it would seem to be a less likely explanation for the results obtained in gerbils where the findings may be entirely attributable to crossing over of the phrenic fibres.

These observations were consistent in the entire series of animals studied irrespective of variations in survival times which were mostly within the range of 4-28 days. Two animals in which the survival times were 153 and 196 days respectively showed many normal argyrophilic fibres in the diaphragm on the side of the lesion. It was considered likely, however, that the vast majority of these were the result of regenerative phenomena rather than representing a persistence of fibres. In the 153-day animal the conspicuous haphazard bundle of fibres which connects with the distal stump of the nerve suggests a diffuse rather than a concentrated single origin for these neuronal processes. The precise origin of these fibres was not located but their direction suggests that they connect with the intercostal nerves laterally and this would be in agreement with the experimental work of Aitken (1950).

The most thought-provoking finding of the entire study was the general irregularity but multisegmental nature of the phrenic nucleus in gerbils as opposed to other mammals in which it is said to occupy a stereotyped position in the anterior horn of two or three cervical segments (Warwick and Mitchell, 1956). It is interesting to speculate regarding the possible explanation of this phenomenon found in two exhaustive studies and subsequently confirmed in another two animals.
The partial allegiance of diaphragmatic function with both somatic and autonomic structurofunctional phenomena is widely recognized in humans. For example, the musculature which can normally be controlled in voluntary apnoea by 'the will' runs amok in diaphragmatic spasm and becomes autonomic in nature. Recent reports indicate that this pathological involuntary function of the diaphragm may exceed 100 contractions per minute (Dressler and Kleinfeld, 1954) and pose a real threat to survival. The extent of the gerbil phrenic nucleus would tend to increase the possible number and hence collective effect of autonomic influences on the motor function of the diaphragm. It is also tempting to suggest that the cells which occupy the most rostral portion of the nucleus may be equivalent to the cells which occupy the intermediolateral grey column of the thoracolumbar region. Whatever the reason may be for this nuclear configuration, it appears to provide good grounds for re-examining the extent of the phrenic nucleus in higher mammals, including man. It may also be worth while to examine further caudally along the spinal cord for morphological evidence to substantiate the findings of Rosenbleuth, Alanis, and Pilar (1961), who were interested in the apparent diffuse nature of spinal centres for respiration.

This study was supported by the Medical Research Council of Canada grant no. MA3436.

\section{REFERENCES}

Aitken, J. T. (1950). Growth of nerve implants in voluntary muscle. J. Anat. (Lond.), 84, 39.

Barstad, J. A. B., Kristoffersen, A., Lilleheil, G., and Staaland, H. (1965). Muscles spindles in the rat diaphragm. Experientia (Basel), 21, 533.

Botha, G. S. M. (1957). The anatomy of phrenic nerve termination and the motor innervation of the diaphragm. Thorax, 12, 50.

Dressler, W., and Kleinfeld, M. (1954). Tic of respiratory muscles; report of three cases and review of literature. Amer. J. Med., 16, 61.

Gairns, F. W. (1930). A modified gold chloride method for the demonstration of nerve endings. Quart. J. micr. Sci., $74,151$.

Garven, H. S. D., and Gairns, F. W. (1952). The silver diamine ion staining of peripheral nerve elements and the interpretation of the results: with a modification of the Bielschowsky-Gros method for frozen sections. Quart.J. exp. Physiol., 37, 131.

Hirako, G. (1928). Uber die Zellsaule des Halsmarks und die Lokalization der Wurzelzellen des Nervus phrenicus der Ratte. Folia anat. jap., 6, 311.

Keswani, N. H., Groat, R. A., and Hollinshead, W. H. (1954). Localization of the phrenic nucleus in the spinal cord of the cat. J. anat. Soc. India, 3, 82. 
Kidd, L. J. (1911). The sensory phrenic and its organs. Rev. Neurol. Psychiat., 9, 587.

Kohnstamm, O. (1898). Zur Anatomie und Physiologie des Phrenicuskernes. Fortschr. Med., 16, 643.

Lanza, G. Giordano (1958). Sulle caratteristiche istologiche delle espansioni nervose sensitive presenti nel diaframma di Macacus rhesus. Boll. Soc. Ital. Biol. sper., 34, 1608.

Rosenblueth, A., Alanis, J., and Pilar, G. (1961). The accessory motor innervation of the diaphragm. Arch. int. Physiol., 69, 19.
Samuel, E. P., and Warwick, R. (1955). The origin of the phrenic nerve in rhesus monkey. J. comp. Neurol., 10 557.

Sherrington, C. S. (1894). On the anatomical constitution $\frac{5}{\mathrm{ff}}$ nerves of skeletal muscles with remarks on recurrent fibres in the ventral spinal nerve-root. J. Physiol. (Londs), 17, 211.

Warwick, R., and Mitchell, G. A. G. (1956). The phrente nucleus of the macaque. J. comp. Neurol., 105, (3), 553.-

Wilson, A. S. (1968). Investigations on the innervation of the diaphragm in cats and rodents. Anat. Rec., 162, 425. 